\title{
Detection of Lung Density Variations With Principal Component Analysis in PET
}

\author{
Ottavia Bertolli, Student Member, IEEE, Vesna Cuplov, Simon Arridge, Charles W Stearns, Fellow, IEEE, \\ Scott D Wollenweber, Senior Member, IEEE, Brian F Hutton, Senior Member, IEEE, \\ Kris Thielemans, Senior Member, IEEE
}

\begin{abstract}
Respiratory motion generates lung volume changes during the breathing cycle. These affect the lung tissue density and therefore influence both the attenuation effect and the radiotracer concentration in PET imaging. To detect and correct for these effects could improve the quantitative accuracy of lung PET imaging. In this work we propose the use of Principal Component Analysis (PCA) to detect respiratory-induced lung density changes in the upper lung, where motion is expected to be minimal. The method is firstly applied to simulation data, specifically generated to simulate density changes only and no motion. Secondly, it is applied on the upper lung bed position of 15 lung cancer patients datasets. The total number of counts in time is also evaluated. The results show that the PCA signal is highly correlated to the respiratory trace obtained from an external device, and also to the variation of total counts in time. As the bed positions taken into account do not include moving organs, the results suggest that PCA is successful in detecting respiratory-induced density changes in the upper lung.
\end{abstract}

Index Terms-PET, PCA, lung density variations

\section{INTRODUCTION}

D URING the breathing cycle the lung density changes as a consequence of the expansion and compression of the chest volume. The changes in density affect the attenuation effect and the observed radionuclide activity concentration in PET imaging, therefore inducing respiratory-related changes in the acquired data both in PET and CT [1].

Detection of the density variations in the lungs can therefore be beneficial when aiming at analysing the respiratory-induced effects on PET data, and furthermore when interested in performing respiratory motion correction, where compensation for density variations could improve the quantitative accuracy of the imaging modality [2].

Principal Component Analysis (PCA) has been shown to successfully detect respiratory motion from the PET raw data

This work was supported by EPSRC Industrial CASE studentship (13220093), co-sponsored by GE Healthcare, and by the National Institute for Health Research, University College London Hospitals Biomedical Research Centre.

O. Bertolli, K. Thielemans and B. F. Hutton are with the Institute of Nuclear Medicine, UCLH, 235 Euston Road (T-5), London, NW1 2BU, UK email: ottavia.bertolli.13@ucl.ac.uk

V. Cuplov was with the Imagerie Moléculaire In Vivo, IMIV, CEA, INSERM CNRS, Université Paris-Sud, Université Paris Saclay, CEA-SHFJ, Orsay, France

S. Arridge is with the Centre for Medical Image Computing, University College London, Gower Street, London, WC1E 6BT, UK

B. Hutton is also with the Centre for Medical Radiation Physics, University of Wollongong, Australia

C. W. Stearns and S. D. Wollenweber are with GE Healthcare, Waukesha, WI, USA.

Manuscript received November 17, 2017. of the chest [3], where the biggest variations is given by the internal motion of the organs. In this work we propose the use of Principal Component Analysis to detect the density variations caused by respiration in the data related to the upper part of the lungs, where motion is minimal and the more significant variation is expected to be caused by lung density changes.

\section{Methods}

\section{A. Simulations}

Density changes will affect both PET tracer concentration and attenuation, with opposite effect on the number of detected counts. Furthermore, as shown in [4], these effects can be estimated by registration between respiratory gates, as the Jacobian determinant of the deformation field is related to local volume changes. We use this here to construct realistic simulated data that incorporate the density changes, by processing patient respiratory gated PET/CT data.

We used one set of patient chest FDG-PET/CT data that was acquired with Cine CT, on a GE Discovery STE scanner. The acquisition was monitored with a Varian ${ }^{\circledR}$ Real-time Position Management ${ }^{\mathrm{TM}}$ device (RPM), the PET and the CT data were gated with displacement gating in 5 gates. The PET gate corresponding to end-inhalation $\left(g_{1}\right)$ was reconstructed with OSEM with attenuation correction performed using the CT gate corresponding to the same breathing phase.

To estimate the local volume change of the lung between gates we registered the CT gated images, as they offer a greater spatial resolution compared to the corresponding PET. Non rigid registration was applied using the NiftyReg software [5], [6] between the end-inhale state and the other 4 gates, and the deformation fields and the related Jacobian determinants were obtained. In order to simulate exclusively the impact of density changes in the lungs on PET data, as opposed to the motion effects, a series of simulated PET gated data was generated: the reconstructed PET gate corresponding to end-inhalation $g_{1}$ (smoothed with an edge-preserving filter to decrease the noise) was multiplied by the Jacobian determinants of the 4 deformation fields, producing a new series of PET gates $g_{2, J}, g_{3, J}, g_{4, J}, g_{5, J}$. The same processing was performed on the attenuation map of $g_{1}$, to generate the matching deformed attenuation maps for each gate. The simulated PET gates were then forward projected using STIR [7] and the generated sinograms were attenuated with attenuation correction factors obtained from the corresponding attenuation map, producing the series of sinograms $s_{2, J}, s_{3, J}$, 
$s_{4, J}, s_{5, J}$. The simulated sinograms were spatially downsampled (as in [3]) and the area related to the upper part of the lungs was selected, by segmenting parts of the sinogram in the radial direction and selecting only Lines of Response through the upper planes, and used for the following analyses.

PCA was then applied to the series of sinograms $s_{g, J}$, leading to the expansion:

$$
s_{g, J} \approx \bar{s}_{J}+\sum_{k=1}^{K} w_{k g} p_{k}
$$

where $\bar{s}_{J}$ is the mean of the sinograms over the gates, $p_{k}$ are the Principal Components (PCs) and $w_{k g}$ their weight factors. The first PC, that is the one corresponding to the biggest variation in the data, was examined together with its weight factor.

To analyse the effect of the attenuation on the forward projections, since the attenuation factors vary between gates as a consequence of the variation in lung density, the total number of counts in each simulated gate was calculated, and the mean value of a Region Of Interest (ROI) in the lung within the sinogram was evaluated, both on sinograms with and without the application of the matching attenuation.

\section{B. Patient data}

PCA was applied on patient PET data of the upper part of the chest, in order to test whether a respiratory-like signal is generated from data related to areas with minimal physical motion. The study was performed on 15 FDG oncology patient datasets acquired in 3D listmode on a GE Discovery 690 PET/CT scanner of 360s duration, monitored by the RPM device. The chosen bed position per patient was corresponding to the upper part of the lungs, which did not include the liver or the diaphragm in the Field Of View (FOV). The listmode files were unlisted into sinograms with a temporal resolution of $500 \mathrm{~ms}$ for the application of PCA, in order to reduce the sensitivity of the method to the cardiac motion. The respiratory PC was selected as in [3]. The PCA signal was subsequently compared to the RPM via evaluation of the Pearson correlation.

In order to investigate more thoroughly the effect of respiratory-induced lung density variations on the detected data (similarly to the ROI and total counts analysis with simulation data), the sum of the total number of counts in each temporal frame of the PET sinogram (after decay correction) was evaluated. The signal given by the variation in detected counts in time was then compared to the RPM signal. The number of detected counts is expected to be directly related to the changes in density of the lungs (and therefore to the changes in attenuation) because the analysed bed positions did not include moving organs such as the liver or the diaphragm, that when present can move in and out of the FOV therefore affecting the detected activity independently of density changes. Note that the number of detected counts was also used in the method presented in [8], where the count rate changes in 3D PET due to organs moving with respect to the centre of the scanner.

\section{RESULTS}

\section{A. Simulations}

The first PC obtained from the application of PCA on the series of simulated sinogram data is shown in Figure 1. On the right-hand side the PC weight factor and the total number of counts are displayed per gate. The diffused features of the PC suggest that the variations detected by PCA are related to local changes of the values in the sinogram, and the trend of weight factor and counts along the gates shows that the effect detected by PCA varies very similarly to the amount of counts during respiration. In Figure 2 the coronal projections without and with the application of the attenuation effect are displayed together with the ROI selected in the upper part of the lung. The relative difference between the mean in the ROI from the gates corresponding to inhalation and exhalation is $-3 \%$ and $+0.5 \%$ on the non attenuated and attenuated data respectively, demonstrating that the changes in density throughout the respiratory cycle have a significant effect on the detected data, and that the effect due to attenuation dominates, at least for this simulation

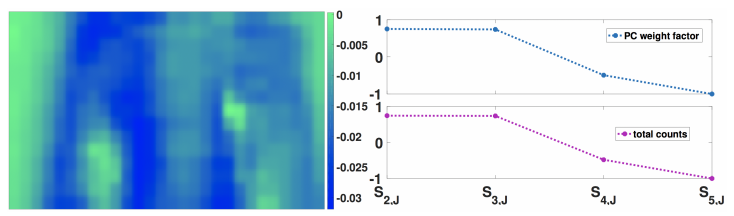

Fig. 1: Coronal view of the first PC. Weight factor and change in total number of counts for each gate.

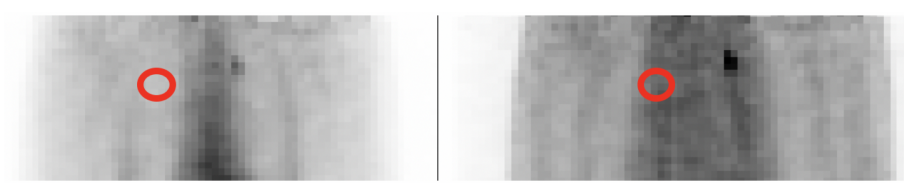

Fig. 2: On the left, the coronal view of the projections of gate 1 without the application of the attenuation effect, on the right the projections with the attenuation effect. In red the selected ROI. Arbitrary grey scale is used in both images.

\section{B. Patient data}

The reconstructed image of a patient dataset is shown in Figure 3. The diaphragm is not included in the FOV. and there was no visible movement in the upper part of the lung.

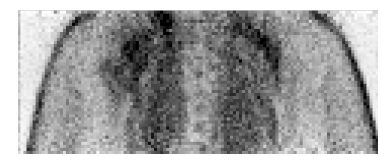

Fig. 3: Example of NAC PET reconstructed image of one patient (coronal projection). The FOV only includes the upper part of the lungs.

In Figure 4 an example of the respiratory signal obtained with PCA is shown together with the signal given by the total number of counts in time and the external device respiratory signal. 
In Table I the mean values over all patients obtained by the comparison with Pearson correlation of the RPM with the PC signal and the total number of counts are shown.

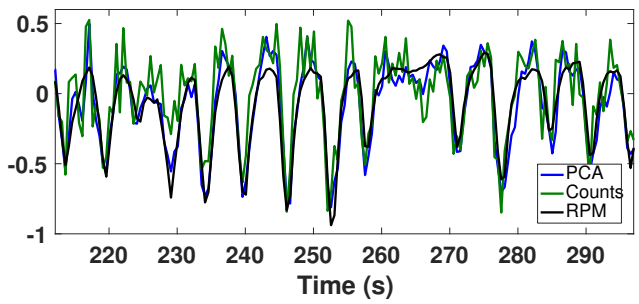

Fig. 4: Signals given by PCA, the sum of the counts and the external device. High similarity between the 3 signals can be observed.

TABLE I: Mean and standard deviation, minimum and maximum values obtained on the 15 patients for the Pearson correlation between respiratory PC and RPM, and between total number of counts and RPM.

\begin{tabular}{l|ccc} 
& mean & $\max$ & $\min$ \\
\cline { 2 - 4 } Corr PCA and RPM & $0.76 \pm 0.17$ & 0.94 & 0.42 \\
Corr counts and RPM & $0.55 \pm 0.15$ & 0.79 & 0.23
\end{tabular}

\section{Discussion}

The results of the application of PCA on the simulations proved that the algorithm is able to detect the respiratory-induced density variations in the data, even if motion is not present. Moreover, due to the associated change in attenuation effect, the detected counts decrease with increasing density, as observed both from the ROI and the total counts evaluation. This effect is reproduced also in the trend of the first PC weight factor.

Furthermore, the application of PCA on patient data of the upper chest (without moving organs in the FOV) generated respiratory signals highly correlated to the RPM signal. Moreover, the total counts per time frame follow a similar trend. These results suggest that the PCA is detecting respiratory-induced density changes in the lung.

While for the upper lung bed positions the total count method gives reasonable results, PCA produces a signal with much higher fidelity to the RPM.

\section{CONCLUSiON}

This work shows that the application of Principal Component Analysis to PET data of the upper lung generates a signal which constitutes a good representation of the respiratory signal, obtained from an external device. Both the simulation and the patient data did not include moving parts of the body, therefore suggesting that the changes detected by PCA are related to density variations. In conclusion, the PCA method is independent of the bed position and gives good results when either density or motion causes changes in the acquired PET data. It is therefore a powerful tool for investigating PET lung acquisitions.
Future work includes the application of the analysis exploiting TOF information (i.e. using TOF sinograms for PCA), and the investigation of the sign-determination problem related to the PCA signal for these bed positions.

\section{ACKNOWLEDGMENT}

We wish to thank François Bernard (BCCA, Vancouver, CA), and Valentino Bettinardi (Scientific Institute Ospedale San Raffaele, Milan, Italy) for the anonymized patient data.

\section{REFERENCES}

[1] V. Cuplov, B. F. Holman, J. R. McClelland, M. Modat, B. F. Hutton, and K. Thielemans, "Issues in quantification of registered respiratory gated PET/CT in the lung," Physics in Medicine and Biology, in press.

[2] B. F. Holman, V. Cuplov, B. F. Hutton, A. M. Groves, and K. Thielemans, "The effect of respiratory induced density variations on non-TOF PET quantitation in the lung," Physics in Medicine and Biology, vol. 61, no. 8, p. 3148, 2016.

[3] K. Thielemans, S. Rathore, F. Engbrant, and P. Razifar, "Device-less gating for PET/CT using PCA," in Nuclear Science Symposium and Medical Imaging Conference (NSS/MIC). IEEE, 2011, pp. 3904-3910.

[4] J. M. Reinhardt, K. Ding, K. Cao, G. E. Christensen, E. A. Hoffman, and S. V. Bodas, "Registration-based estimates of local lung tissue expansion compared to xenon CT measures of specific ventilation," Medical image analysis, vol. 12, no. 6, pp. 752-763, 2008.

[5] S. Ourselin, A. Roche, G. Subsol, X. Pennec, and N. Ayache, "Reconstructing a 3D structure from serial histological sections," Image and vision computing, vol. 19 , no. 1, pp. 25-31, 2001.

[6] M. Modat, G. R. Ridgway, Z. A. Taylor, M. Lehmann, J. Barnes, D. J. Hawkes, N. C. Fox, and S. Ourselin, "Fast free-form deformation using graphics processing units," Computer methods and programs in biomedicine, vol. 98 , no. 3, pp. 278-284, 2010.

[7] K. Thielemans, C. Tsoumpas, S. Mustafovic, T. Beisel, P. Aguiar, N. Dikaios, and M. W. Jacobson, "STIR: software for tomographic image reconstruction release 2," Phys. Med. Biol., vol. 57, no. 4, p. 867, 2012.

[8] J. He, G. J. O'Keefe, S. J. Gong, G. Jones, T. Saunder, A. M. Scott, and M. Geso, "A novel method for respiratory motion gated with geometric sensitivity of the scanner in 3d pet," IEEE Transactions on Nuclear Science, vol. 55, no. 5, pp. 2557-2565, 2008. 